\title{
A multi-modal discrete-event simulation model for military deployment
}

\author{
Uğur Z. Yıldırım*, Barbaros Ç. Tansel, İhsan Sabuncuoğlu \\ Department of Industrial Engineering, Bilkent University, Bilkent 06800, Ankara, Turkey
}

\section{A R T I C L E I N F O}

\section{Article history:}

Received 23 February 2008

Received in revised form 5 June 2008

Accepted 27 September 2008

Available online 1 November 2008

\section{Keywords:}

Discrete-event simulation

Military deployment

Event graphs

Java

Geographical information system

\begin{abstract}
A B S T R A C T
This paper introduces a logistics and transportation simulation that can be used to provide insights into potential outcomes of proposed military deployment plans. More specifically, we model a large-scale real-world military deployment planning problem (DPP) that involves planning the movement of military units from their home bases to their final destinations using different transportation assets on a multi-modal transportation network. We apply, for the first time, the event graph methodology and listener event graph object framework to create a simulation model of the DPP. We use and extend Simkit, an open-source Java Application Programming Interface for creating discrete-event simulation (DES) models. We use a medium-resolution modeling approach, as opposed to either high-resolution or low-resolution modeling paradigms, to reduce lengths of simulation runs without compromising reality. To accurately incorporate real and detailed transportation network data into the simulation, we use GeoKIT, a licensed, state-of-the-art, Java-based geographical information system. While our DES model is not a panacea for all, it allows for testing the feasibility and sensitivity of deployment plans under stochastic conditions prior to committing members of the military into harm's way. The purpose of the paper is to acquaint the readers with the details of the DPP, the simulation model created, and the results of the analysis of a typical real-world case study.
\end{abstract}

(c) 2008 Elsevier B.V. All rights reserved.

\section{Introduction}

Regional and asymmetric threats and the increase in worldwide terrorist activity have made logistics and mobility increasingly important in our rapidly changing world. This paper focuses on logistics and transportation simulations or computer-based planning tools that are used to provide insight into the potential outcomes of proposed logistical courses of actions prior to and after committing members of the military into harm's way. Specifically, we deal with the Deployment Planning Problem (DPP), defined and thoroughly described first by Akgün and Tansel [1]. DPP involves positioning of many military units to carry out a mission. During peace-time, military units move from their home bases to their designated destinations using different transportation assets. This movement usually takes place on a multi-modal (land, rail, sea, air, and inland waterways) transportation network. During a crisis, where time is of essence, it has become critical to move soldiers and equipment with limited resources and on a short notice. The movement of the units must conform to a preplanned timetable called time-phased force deployment data (TPFDD). The TPFDD describes, among other things, the initial departure times of military units from their home bases, and their earliest and latest arrival times at their designated destinations. When many units need to deploy, the TPFDD is intended to coordinate their movement in order to efficiently use the existing transportation assets and network. It is also meant to prevent congestion at destinations and transfer points, where mode changes are necessary. Yet, creating TPFDD requires joint work of well-trained logistical and operational planners, and is

\footnotetext{
* Corresponding author. Tel.: +90 312 4112773; fax: +90 3122664054.

E-mail addresses: uziya@bilkent.edu.tr (U.Z. Yıldırım), barbaros@bilkent.edu.tr (B.Ç. Tansel), sabun@bilkent.edu.tr (İ. Sabuncuoğlu).
} 
very time consuming. Military deployment planners need a fast and accurate tool that takes into account the stochastic nature of events to analyze a military deployment plan.

A deployment plan may not always go as initially planned. Unexpected breakdown of transportation assets, road traffic accidents, and congestion at transfer points are some of the things that may disrupt a plan. A deployment involves simultaneous movement and utilization of many entities, resources and transportation assets. Thus, a stochastic model is more suitable for this truly hard and real-world problem that deals with expensive military hardware and irreplaceable human life.

In addition, existing models and simulations are of varying resolutions. Most of the time, higher-resolution models provide input to the lower-resolution models. This makes it necessary to run several models in succession for analysis. But such a set-up takes a long time. Almost all of the deployment models require a specific hardware system to run on. Yet, military usually employs different hardware systems, and thus it would be useful to have models or simulations which can run on multiple platforms. A detailed and accurate representation of the transportation network and infrastructure is necessary for realistic analysis. Thus, there is a need for a geographical information system to be used with deployment models or simulations. Currently existing models or simulations either do not have this capability or have a limited representation of geographical information. Furthermore, not all transportation modes are modeled in all deployment models or simulations, which makes it necessary to run at least two models in succession for a large deployment scenario. This again increases set-up and run times. Thus, it is desirable to have a multi-modal simulation model.

For these reasons, we have decided to develop a multi-modal, platform-independent, discrete-event simulation model of military deployment with accurate transportation network and infrastructure data and a medium-resolution allowing planners to develop and analyze plans in a relatively short time.

\section{Background}

There exist deployment planning models and simulations with varying levels of detail and purpose. For a more comprehensive survey of military planning systems and a review of strategic mobility models supporting the defense transportation system, the interested reader is referred to Boukhtouta et al. [2] and McKinzie and Barnes [3]. It is possible to classify military deployment models and simulations into two groups depending on their level of resolution and the purpose of use. First group includes relatively low-resolution models and simulations that may be used to model deployment of military units between theaters of operation (e.g., from Turkey to Afghanistan) or inside a theater of operation (e.g., inside Turkey). Deployments between different theaters of operation using only air and sea transportation assets are referred to as strategic deployment. The most frequently used modeling tools (software) for modeling strategic deployments are NATO's ADAMS (Allied Deployment and Movement System) and U.S. Military's JFAST (The Joint Flow and Analysis System for Transportation). A technical guide for ADAMS is provided by Heal and Garnett [4] and general information for JFAST is available at http://www.jfast.org. An example of simulations modeling deployment inside a theater of operation is ELIST (The Enhanced Logistics Intra-Theater Support Tool) [5]. The second group includes higher-resolution models and simulations that may also be used to provide input to the models in the first group. Examples of these models and simulations are TLoaDS (The Tactical Logistics Distribution System) [6] and PORTSIM (The Port Simulation) [7]. Other important examples of such models are TRANSCAP [8], SIMULOGS [9], and Simulation

Table 1

Mobility planning, logistics and transportation models and simulations commonly used by military planners.

\begin{tabular}{|c|c|c|c|c|c|c|}
\hline Simulations/models & $\begin{array}{l}\text { Multi-modal } \\
\text { (yes/no) }\end{array}$ & $\begin{array}{l}\text { Air }(A) \text {, rail }(R), \text { sea } \\
(S) \text {, land }(L)\end{array}$ & $\begin{array}{l}\text { Platform } \\
\text { independence } \\
\text { (yes/no) }\end{array}$ & $\begin{array}{l}\text { GIS- } \\
\text { support }\end{array}$ & $\begin{array}{l}\text { Discrete-event } \\
\text { simulation } \\
\text { (yes/no) }\end{array}$ & Comments \\
\hline ADAMS [4] & Yes & $A, S$ & No & Limited & No & $\begin{array}{l}\text { A NATO model for strategic } \\
\text { deployment }\end{array}$ \\
\hline JFAST & Yes & $A, S$ & Yes & Limited & No & $\begin{array}{l}\text { A classified, joint US model } \\
\text { for strategic deployment }\end{array}$ \\
\hline ELIST [5] & Yes & $\mathrm{A}, \mathrm{R}, \mathrm{S}, \mathrm{L}$ & Yes & Limited & Yes & $\begin{array}{l}\text { For intra-theater support } \\
\text { planning }\end{array}$ \\
\hline TLoaDS [6] & Yes & $\mathrm{A}, \mathrm{R}, \mathrm{S}, \mathrm{L}$ & No & Limited & Yes & $\begin{array}{l}\text { Built using EXTEND }{ }^{\mathrm{TM}} \text { and } \\
\text { SDl Industry Pro }\end{array}$ \\
\hline PORTSIM [7] & Yes & $\mathrm{R}, \mathrm{S}, \mathrm{L}$ & No & Limited & No & $\begin{array}{l}\text { Simulates seaport } \\
\text { operations and determines } \\
\text { throughput at the port }\end{array}$ \\
\hline TRANSCAP [8] & Yes & $\mathrm{L}, \mathrm{R}$ & Partially & No & Yes & $\begin{array}{l}\text { Models deployment from US } \\
\text { Army installations }\end{array}$ \\
\hline SIMULOGS [9] & No & $\mathrm{L}$ & Partially & Limited & Yes & $\begin{array}{l}\text { Runs on Sun Unix } \\
\text { Workstation and PCs with } \\
\text { Windows NT }\end{array}$ \\
\hline $\begin{array}{l}\text { Simulation of } \\
\text { transportation logistics } \\
{[10]}\end{array}$ & Yes & $\mathrm{A}, \mathrm{S}$ & No & No & Yes & $\begin{array}{l}\text { Based on ARENA, uses VBA } \\
\text { and Excel }\end{array}$ \\
\hline $\begin{array}{l}\text { The simulation model } \\
\text { proposed in this paper }\end{array}$ & Yes & A, R, S, L & Yes & Yes & Yes & $\begin{array}{l}\text { Written in Java and uses } \\
\text { GeoKIT for GIS-support }\end{array}$ \\
\hline
\end{tabular}


of Transportation Logistics [10]. Table 1 gives a comparison of the aforementioned models in terms of their characteristics for multi-modality, platform independence, GIS-support, and discrete-event simulation modeling capability.

None of the models or simulations relevant to this study in Table 1 meets all of these five criteria fully. Our proposed simulation model is listed at the bottom of Table 1 . We have developed a simulation model of military deployment that meets all of these criteria and has a "medium-resolution". More specifically, the modeling level of detail at transfer points for infrastructure is low-resolution. That is, we use aggregate capacities for each individual transfer point and time delays at these locations for problems such as maintenance breakdowns etc. For modeling transportation assets, cargo and resources, we use high-resolution modeling which includes specific dimensions of cargo, and capacities, speeds, and dimensions of resources and transportation assets. Furthermore, the GIS data is of high-resolution, and it specifies, among other things, the capacities and classifications of bridges, roads and other detailed data of related transportation infrastructure.

Our medium-resolution approach to modeling the DPP allows us to obtain quick "first-cut" insights into potential outcomes of a deployment plan without compromising reality. For example, for a typical deployment scenario for four battalions, run times are between 25 and 27 min for 60 runs of the model on a $1.6 \mathrm{GHz}$ Pentium(R) M PC with 512 MB RAM. That is less than $30 \mathrm{~s}$ per run. While our medium-resolution simulation model is not a panacea for all, it provides a realistic and quick litmus test for the applicability of existing deployment plans and allows a quick construction of contingency deployment plans. It is an all-encompassing model for all modes of transportation. Yet, our model's extendable architecture is flexible enough to accommodate future addition of higher-resolution sub-modules intended especially for detailed modeling at transfer points. Moreover, our model is generic enough to be used in commercial logistics applications after some problemspecific modifications.

This study is intended to eventually become part of a capability planning system being developed for the Scientific Decision Support Center of the Turkish General Staff Headquarters. Our simulation model will also be used along with a comprehensive optimization model of the DPP [1] developed as part of the capability planning system mentioned above.

In Section 3, the DPP is explained in detail. The simulation model of deployment problem is presented in Section 4, where conceptual and logical models are explained, and verification and validation issues (V\&V) of our model are discussed. A real and typical case-study scenario is explained and analyzed in Section 5. Conclusions are presented in Section 6.

\section{Problem and system definition}

The DPP deals with the movement of many military units from their Areas of Responsibilities (home bases) to their Tactical Assembly Areas (final destinations). The movement could be either an intra-theater or an inter-theater type. Intra-theater movement can be regarded as the movement of units using different modes of transportation (e.g., land, sea, air, and rail) inside a country's borders. Inter-theater movement refers to the movement of units between countries using air and sea assets (strategic deployment). Once the units reach the destination country, then other available modes of transportation can be utilized inside that country. In this context, the terms "theater" and "country" are used synonymously.

During intra-theater movement, a unit may go directly from its home base to its final destination throughout the entire journey using a single mode of transportation assets (TAs) on a given mode of transportation network that supports the movement of the TAs under consideration. It may also use in succession any of land, rail, sea, or air transportation networks and the TAs dedicated to them, making mode changes as necessary along the way. However, the fewer the mode changes are at transfer points, the easier is the deployment. If a transfer is necessary, the initial movement from home bases is by ground transportation to a transfer point (a location where the movement switches from one mode of transportation to another). Main transfer points are harbors, train stations, and airports. At these locations, the pax (troops) and cargo (weapon systems, material, equipment, and supplies) of a unit are transferred from one set of TAs to another set that operate on a different network. This location is also called a Port of Embarkation (POE). The next mode change location, where the items are offloaded and loaded onto another set of TAs is called a Port of Debarkation (POD). These may be sea, rail, and air POEs or PODs. Inter-theater movement differs from intra-theater movement only by its use of strategic lift (air and sea) assets to reach the next theater of operations.

At a transfer point, units are held in a staging area to prepare for shipment before being loaded on vessels. However, in many cases, there is not enough room at a terminal to stage the entire unit or many units scheduled to move at the same time. In such cases, a marshaling area is operated. Marshaling area provides a location to receive unit personnel, equipment, and supplies, and prepare them for movement prior to entering the staging area. As the transport vessel (e.g. a ship, a train or an airplane) gets ready, the units are called from the marshaling area to the staging area. The two areas serve much the same purpose. A staging area can be regarded as a service point, i.e. one with a certain capacity of material handling equipment and load/unload docks, and a marshaling area as a waiting/parking place. They help provide an uninterrupted flow of items through their transfer points. Staging/marshaling areas are also operated at home bases and destinations [1].

A unit may be divided into three components (forward party, pax party, and cargo party) during deployment. Ground movement is usually conducted in convoys to maintain the unity of the component, and the size of the convoys may vary depending on operational/tactical objectives and limitations. The synchronization of departures of these components from their home bases and their arrival at their designated destinations is dictated by operational requirements, threat level, availability and capacity (lanemeter, seat, volume, weight) of lift assets, and the current conditions of transportation infrastructure [1]. 
A unit will usually use its own (organic) TAs to conduct a deployment. However, for heavy lift requirements (for example tanks and artillery pieces) over long distances, TAs of other military transportation units may have to be used. In addition, outsourcing of TAs from national civilian companies or other nations may be required depending on the distances and numbers and sizes of units involved in the deployment.

While time is of essence during a crisis, cost may be of main concern during peace-time. The source of TAs used affects the cost and timing issues of unit movements. For example, outsourced TAs may not be available on-time and leasing costs are associated with them. In addition, unpredictable stochastic events (breakdowns, accidents, delays etc.), load/unload/idle times at home bases/destinations/transfer points, convoy speeds, and speeds of transportation assets need to be taken into account to determine if a plan of deployment may be realized in actuality.

The planning for a particular deployment may take place beforehand. This is called deliberate planning when time is not a critical factor. When the time available for planning for actual deployment of armed forces is short, this is called crisis planning or time-sensitive planning where the planning process must be quick and flexible to adapt to changing situations. Deliberate planning may contribute to time-sensitive planning. Whether deliberate or not, each deployment plan has a TPFDD which at least includes units' transportation requirements by type and quantity, and movement data by mode, earliest times of departures from home bases, and earliest and latest times of arrivals at POEs/PODs/destinations. It divides a unit's components by transportation mode, ports of embarkation or debarkation, and movement dates.

\section{The discrete-event simulation model}

We used the event graph (EG) methodology developed by Schruben [11] and improved by Sargent [12] and Buss [13] to represent the conceptual and logical models of our simulation. In the EG methodology, nodes represent events and directed arcs represent the scheduling relationships between events. A tilde in an arc represents the conditional scheduling of the event at the head of the arc whenever the stated Boolean condition is satisfied. Dashed arcs represent canceling relationships. A special event, referred to as "Run", is used to initially populate the event list as well as to initialize the state variables. In a given module, the Run event may be connected to another event via a directed arc or may appear as an isolated event. In the former case, it performs both of its functions (i.e., scheduling the initial event as well as initializing the state variables). In the latter case, the only function performed by the Run event is initialization of the state variables. EGs can be used to, simply and elegantly, represent any DES model.

LEGO framework [14] is used to create components of our simulation. LEGOs are an extension to basic EGs which allow small models to be encapsulated in reusable modules. These modules can be treated as components of other modules. This modular structure is depicted by drawing a box or rectangle around the EG. Modules or components are linked using the listener pattern of Object-Oriented Programming which enables production of larger and more complex modules. LEGOs register interest in other LEGOs and take appropriate actions when they "hear" state changes. The LEGO that is listened to is not affected and is not responsible for any actions taken. This connection is enabled by having events with the same name and signature in both components. This "listener" and "listened" relationship is depicted via an arc with a reversed triangle at one end, resembling a stethoscope. The object at the end of the arc with the reversed triangle is the "listened" object as depicted in Fig. 1.

This loose-coupling of objects in the simulation allows a great amount of flexibility. EGs and LEGOs can be programmed using Simkit, a Java Application Programming Interface (API) developed at the Naval Postgraduate School and freely available via http://diana.nps.edu/Simkit/. Simkit has also been selected as the simulation engine for Combat XXI, the US Army's nextgeneration premier ground combat simulation. It integrates object-oriented design with a listener pattern to create a truly reusable component architecture for model components. The listener pattern implementation in Simkit is called Simulation Event Listener or "SimEventListener" pattern using Simkit's interface name [14,15]. Simkit, utilizing the same ideas of listener patterns, has a very loose-coupling of model components. This permits a clean separation of model constructs from data gathering. Simkit's use of the "PropertyChangeListener" pattern for collecting statistics from a simulation model ensures that any Measures of Effectiveness (MOEs) can be estimated.

To satisfy the requirement for accurate animation based on real transportation network data (such as the capacities of roads, railways and bridges), our simulation model uses a state-of-the-art Java $2^{\mathrm{TM}}$ based, licensed geographical information system (GIS) named GeoKIT, as part of the transportation simulation developed. GeoKIT is an API for manipulating and visualizing $2 \mathrm{D} / 3 \mathrm{D}$ raster and vector spatial data. It is written in the Java ${ }^{\mathrm{TM}}$ programming language and provides a comprehensive set of components to embed GIS functionality into the applications. GeoKIT is open to all types of geographical data and is independent of any particular data format. It achieves high performance mapping and precise geodetic calculations, coordinate transformations and map projections. With its object-oriented design in Java, it allows an intrinsically perfect interaction with Simkit and the EG paradigm. GeoKIT and the available GIS data allow high-resolution representation of transportation infrastructure (e.g., roads, railroads, bridge capacities, slope information, etc.). Using Java as the programming language provides an advantage due to its portability across the multiple platforms employed by military analysts. Furthermore, using the object-oriented paradigm for model development fosters the greatest amount of modularity and potential for reuse for future extensions to our simulation. More information on GeoKIT can be found at http://geokit.bilgigis.com.

Our simulation model, developed using the tools briefly described above, has three main components; graphical user interface (GUI), network, and model. The GUI component allows, among other things, an accurate animation using real geospatial 


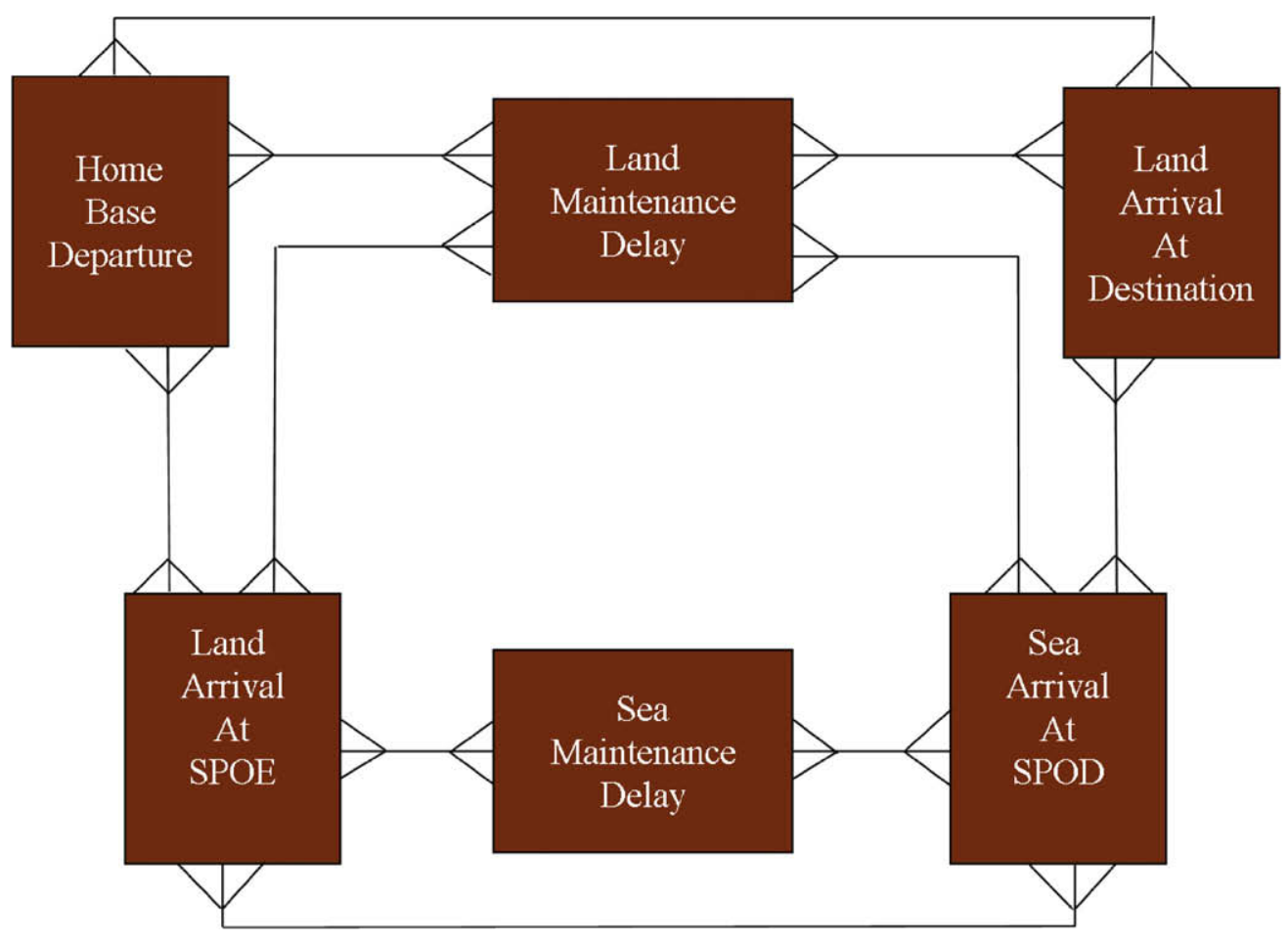

Fig. 1. The SimEventListener pattern for land and sea components.

transportation infrastructure data, point-and-click operations of route selection, adding/deleting/changing home bases/destinations/POEs/ PODs and entities, and on-the-fly projection of the entire network from World Geodetic System (WGS) 84 to Universal Transverse Mercator (UTM) coordinates. WGS 84, developed in 1984 and updated in 2004, is an ellipsoidal reference frame for the earth for use in geodesy and navigation. UTM coordinate system was developed by NATO in 1947 and is used by most military maps in the world. The scenario information can be saved in XML format.

The network component allows shortest path selection in route planning. In addition, it allows the listing and selection of all routes on land and rail networks whose length (cost) is a user-specified percentage more than the shortest path's.

The model component has four components: land, sea, rail, and air. Each of these has three subcomponents. The subcomponents are connected via LEGOs and SimEventListener frameworks.

In addition, there are components, or Java classes, modeling the transportation assets (e.g., trucks, ships, airplanes) of different capacities and modes. The loads of different sizes and military hardware that need to be transported (such as tanks, generators, and field artillery guns) are also modeled. These do not exist in Simkit and have been added as extensions.

\subsection{SimEventListener pattern for land and sea components}

The land and sea components and their subcomponents are connected as depicted in Fig. 1.

A unit departing its home base may directly go to its destination or arrive at a Sea Port of Embarkation (SPOE) to load its items onto a ship. After arrival by sea at a Sea Port of Debarkation (SPOD), the items will be unloaded and the unit will travel by land to its destination. Every time land transportation assets are used, maintenance delays may occur. These are modeled in the land maintenance delay subcomponent. The delays due to breakdown of equipment and road traffic accidents are taken into account and the arrival time of the unit at its destination is updated accordingly. A similar situation is true for sea transportation assets departing a SPOE and arriving at a SPOD and vice versa. In order for two subcomponents to listen to each other, they both need to have events with the same name and signature. The same listener pattern explained above is true for SimEventListener pattern for land and rail components and the SimEventListener pattern for land and air components.

\subsection{Home base departure subcomponent}

Home base departure subcomponent simulates the loading of vehicles at their home bases, forming of convoys and their departure. The movements are conducted in convoys either to the destination or the next mode change location (transfer point) which can be a SPOE, a RPOE, or an APOE. Vehicles that do not need to make additional trips simply park at their home bases. The simplified event graph for this class is in Fig. 2. 


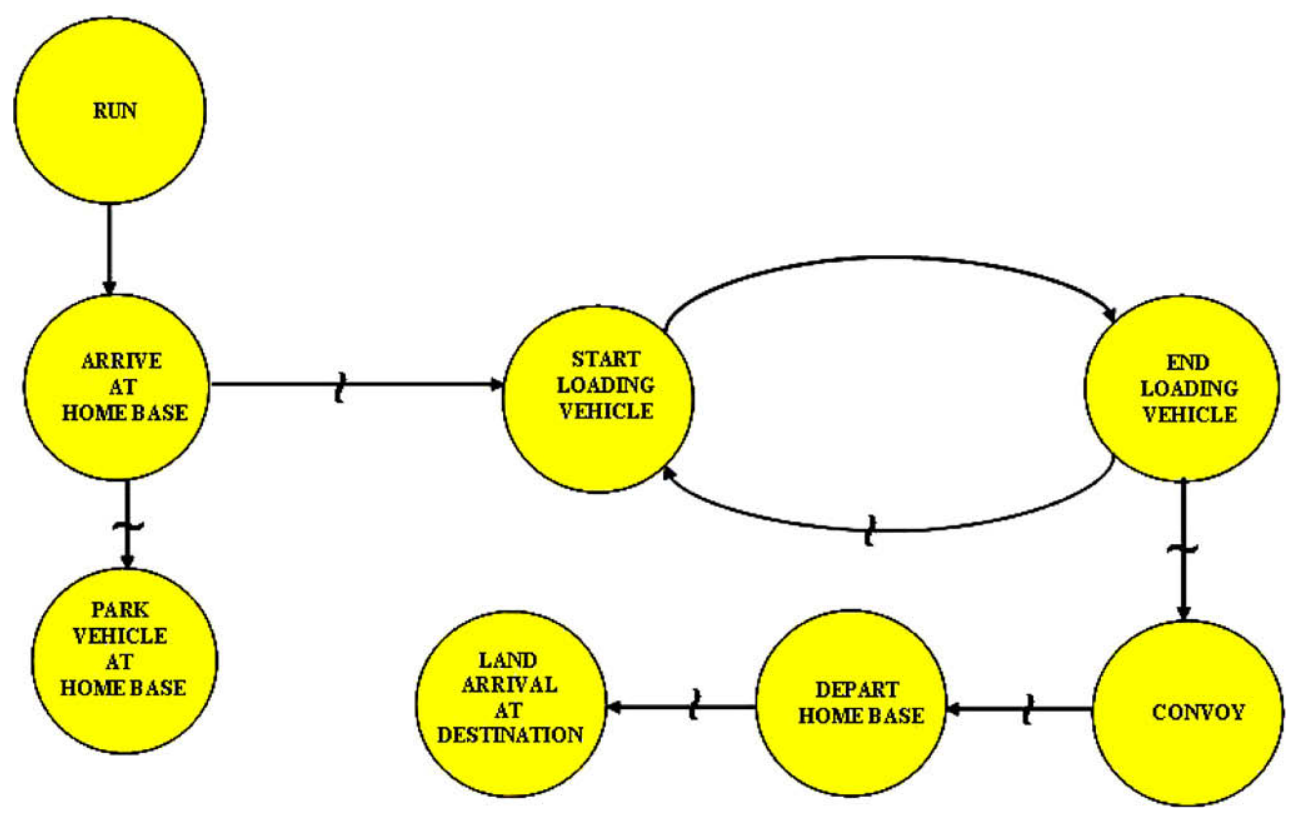

Fig. 2. Simplified event graph for home base departure subcomponent.

Note that the arrival events at SPOE, RPOE and APOE are omitted from this event graph to keep it simple for presentation purposes. The Run event in Fig. 2 is used to schedule the first events (initial population of the event list) such as assigning movers (trucks, ships, trains, etc.) to their respective initial locations and also to specify earliest times of departures for the movers from their home bases. All arcs in Fig. 2 except two of them are marked by a tilde indicating that the events at the head of the marked arcs are scheduled conditionally. For example, the arc connecting "ArriveAtHomeBase" to "StartLoadingVehicle" schedules the event "StartLoadingVehicle" in the event list only if the Boolean condition "number of load docks available $>0$ " is satisfied. The Boolean conditions are omitted from the figure for the sake of simplicity. We also omit the parameters and state variables from the figure for the same reason. These are presented for a specific scenario in the case study in Section 5.

\subsection{Land maintenance delay subcomponent}

Land maintenance delay subcomponent simulates possible causes of delay that may take place during the land movement of a convoy from its home base to its destination. In addition, the delays that may occur during land movement from the transfer points SPOE, APOE or RPOE (SPOD, APOD, or RPOD) to the home base (destination) are also taken into account in the same class. The simplified event graph for this class is provided in Fig. 3. The possible causes of maintenance delay are minor, medium, severe breakdowns in land transportation assets and road traffic accidents. Only minor breakdown case during travel from a home base to a destination is depicted in Fig. 3.

The probabilities of breakdown are different for each type of land vehicle and they are obtained from the Turkish Army Logistics Directorate. The most probable locations of road traffic accidents are determined according to the historical data of so-called "black spots" on Turkey's roads. The data is obtained from http://www.kgm.gov.tr/asps/trafik/karanokta.htm and incorporated into the GIS. The probabilities of traffic accidents for these locations are obtained from a report prepared by the Turkish General Directorate of Highways for the years 1997-2002.

The simplified event graph in Fig. 3 states that if a minor breakdown occurs during travel from a home base to a destination, then the arrival event to destination is canceled (the dashed arc) and after a delay time of maintenance obtained from a probability distribution, the arrival at destination is rescheduled. In a similar way, there are also possibilities of additional types of breakdown (medium and severe) and accidents when a convoy is on its way from its home base to its destination, or when it is on its way from an intermediate location such as SPOE, APOE or RPOE (SPOD, APOD, or RPOD) to its home base (destination). Certainly, there is the possibility of not having any problems while en route, and it is also incorporated into our model. Anytime land transportation assets are used, breakdowns and road traffic accidents are possible to occur. However, the events of LandArrivalAtSPOE(RPOE,APOE), LandArrivalAtSPOD(RPOD,APOD), DepartSPOE(RPOE,APOE), DepartSPOD(RPOD,APOD), DepartDestination, ArriveAtHomeBase, Medium(Major) Breakdown, RoadAccident and the canceling arcs for LandArrivalAtSPOE(RPOE,APOE), LandArrivalAtSPOD(RPOD,APOD) and ArriveAtHomeBase are omitted from Fig. 3 to keep the presentation simple. 


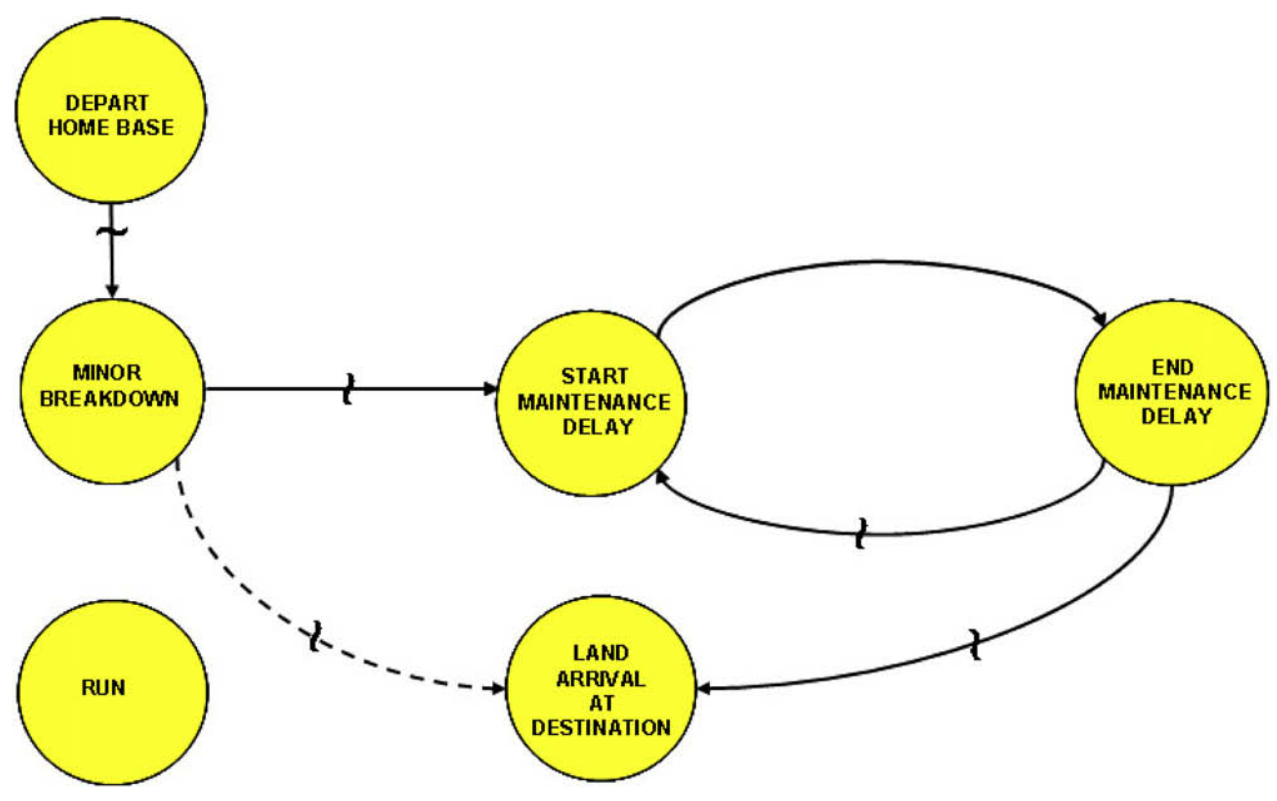

Fig. 3. Simplified event graph for land maintenance delay subcomponent.

\subsection{Land arrival destination subcomponent}

Land arrival destination subcomponent simulates the arrivals of convoys at their designated destination locations, unloading of the vehicles at destinations and forming of convoys and their departures to bring the remaining items of the unit (from their designated home bases, SPODs, RPODs or APODs) if additional trips are required. Otherwise, the vehicles park and stay at their destination locations until a next order for movement is given. The vehicles will also park and stay there upon reaching the destination if they are not carriers (e.g. tanks). The event graph for the land arrival destination subcomponent is provided in Fig. 4. The VehicleArrivalAtSPOD(RPOD, APOD) events scheduled by DepartDestination event are not included in Fig. 4 to keep the presentation simple.

\subsection{Land arrival at SPOE subcomponent}

The land arrival at SPOE subcomponent simulates the arrival of a land convoy at its transfer point, SPOE, for loading into a sea vessel. The vehicles of the convoy along with their loads can be directly loaded into the sea vessel if it is of RoRo type (Roll-on Roll-off, i.e., ferries designed to carry wheeled cargo such as cars, trucks etc.). Otherwise, the items have to be unloaded from the vehicles and then loaded into the sea vessel. If the ship type is not of RoRo and thus the vehicles do not go with the ship, then the unloaded vehicles may be loaded (if any loadable items are at the port) and depart for their next destination. They may also depart the SPOE empty and go to their next destination or original location (home base) if a return trip is required. These events are described in the event graph in Fig. 5.

\subsection{Sea maintenance delay subcomponent}

Sea maintenance delay class simulates possible different causes of delay that may take place during the movement of a sea vessel from its SPOE to SPOD and vice versa. The information on different causes of delay and maintenance times for sea vessels simulated were obtained through talks with experts at the Turkish Navy Command Headquarters.

Propeller breakdowns and steer breakdowns are more likely to occur but they can be fixed by the personnel aboard the ship. These are classified as minor breakdowns. On the other hand, gyro main machine and radar breakdowns are less likely to occur but they cannot be repaired by the personnel aboard. These types of breakdowns would require the towing of the ship to a nearest port where repair facilities exist. These are classified as major breakdowns. Certainly, there is the possibility of departing the SPOE (SPOD) and arriving at the SPOD (SPOE) without any problems while en route. The event graph for this class is in Fig. 6.

Note that only the minor breakdown case is depicted in Fig. 6 to keep the presentation simple. The simplified event graph in Fig. 6 states that if a minor breakdown occurs during travel from a SPOE (SPOD) to a SPOD (SPOE), then the arrival event to SPOD (SPOE) is canceled (the dashed arc) and after a delay time of maintenance obtained from a probability distribution, the arrival at SPOD (SPOE) is rescheduled. 


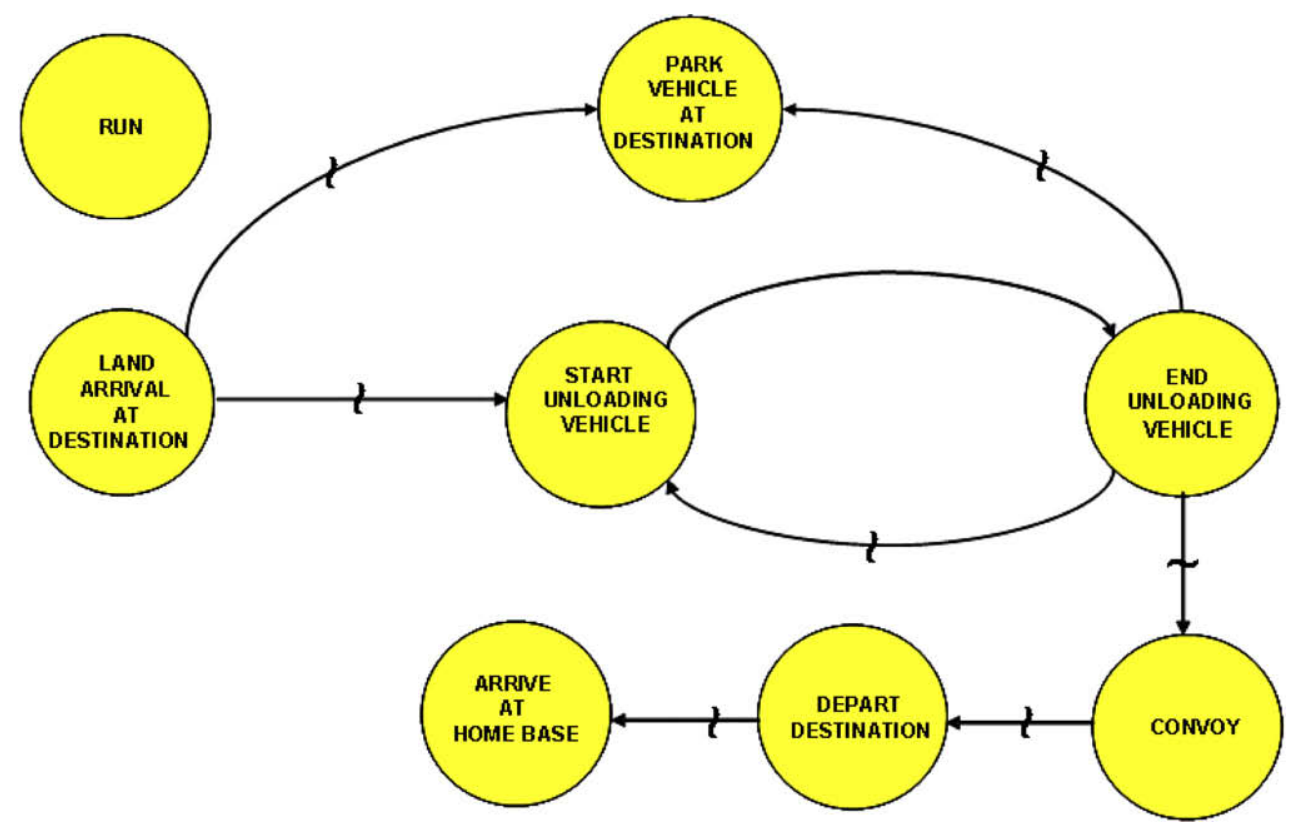

Fig. 4. Simplified event graph for land arrival destination subcomponent.

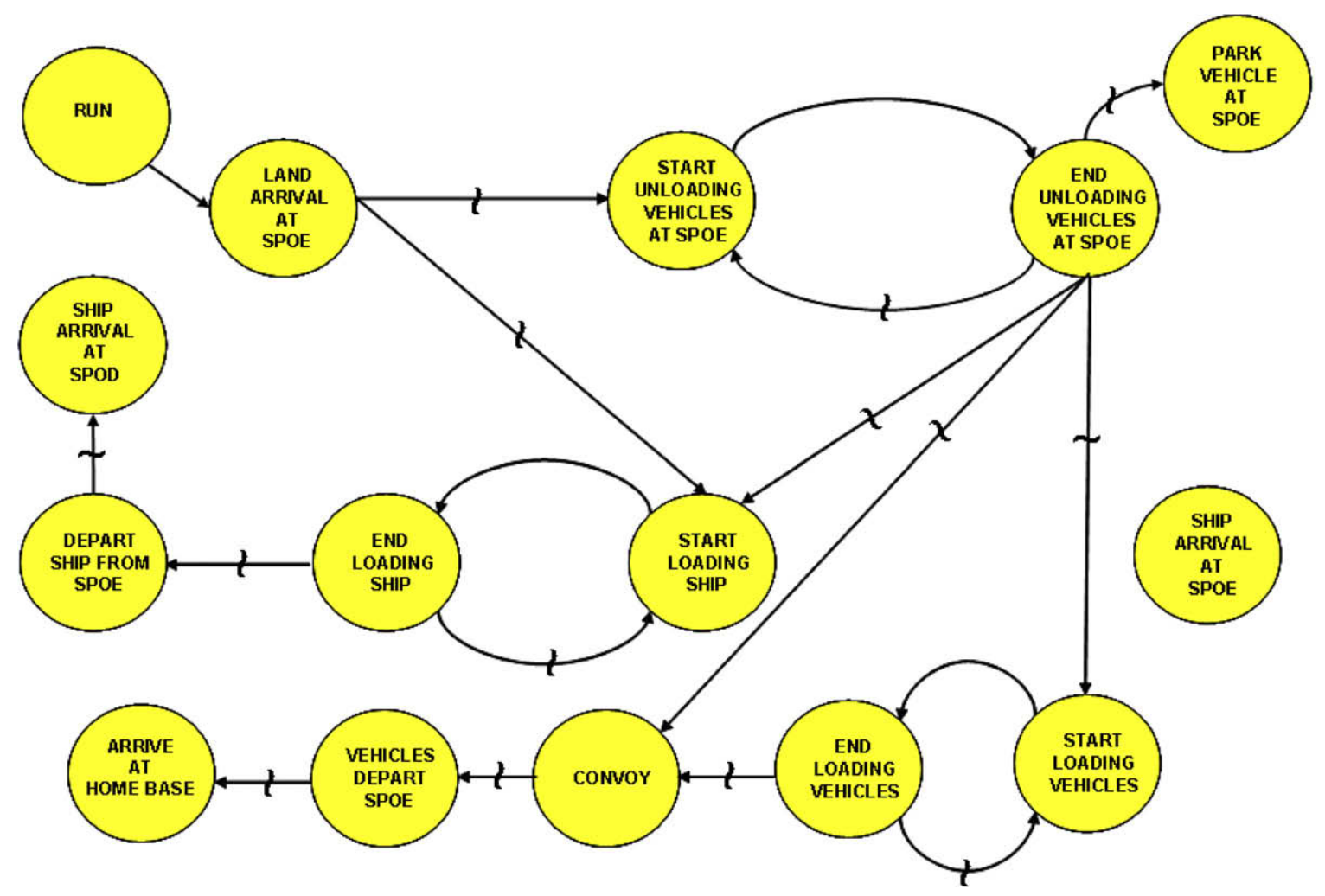

Fig. 5. Simplified event graph for land arrival at SPOE subcomponent.

\subsection{Sea arrival at SPOD subcomponent}

Sea arrival at SPOD subcomponent simulates the arrival of a ship at its SPOD and unloading of the ship. If the ship is of RoRo type, then the vehicles that debark may immediately form convoys and depart the SPOD for their destination. If the 


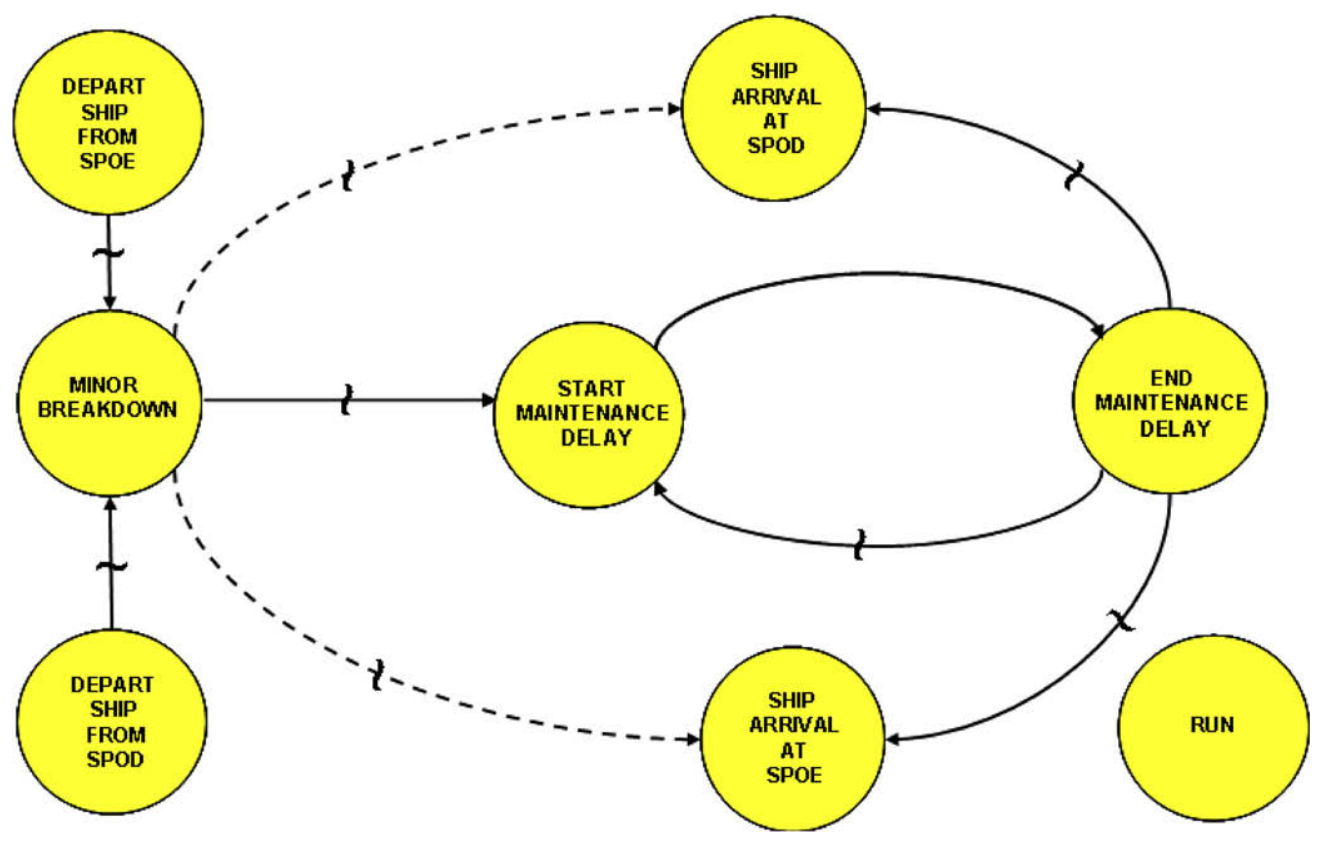

Fig. 6. Simplified event graph for sea maintenance delay subcomponent.

ship is not of RoRo type, then the items have to be unloaded from the cargo ship, loaded onto vehicles present at the port for transporting the unloaded items. Then convoys have to be formed and depart for their destinations. The ship may immediately leave after discharging or sail to its SPOE after loading any available items if a return trip is required or if the ship is ordered to return. The simplified event graph for this class is in Fig. 7. The event graphs for subcomponents of air and rail components are not included in this paper.

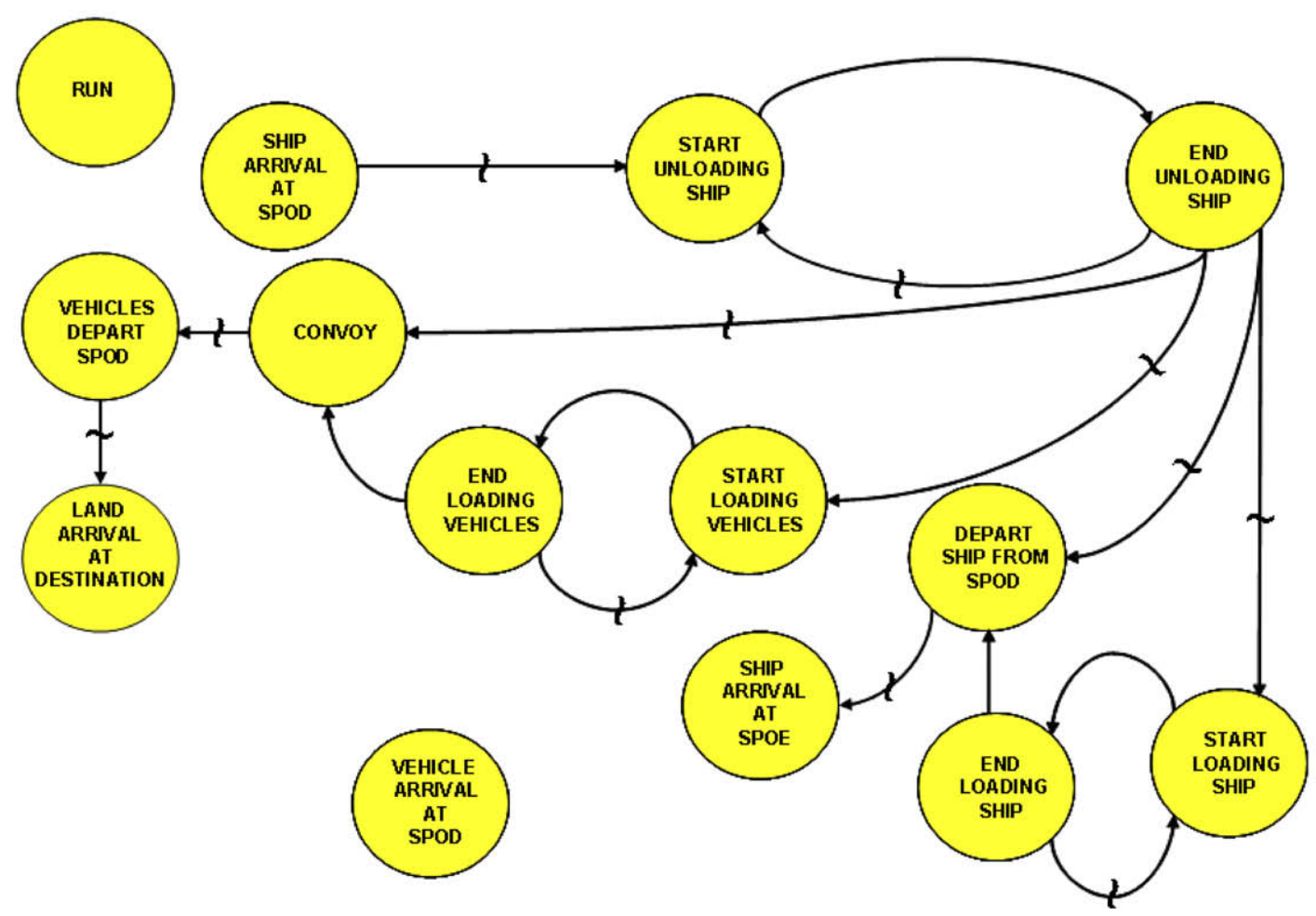

Fig. 7. Simplified event graph for sea arrival at SPOD subcomponent. 


\subsection{Animation and graphical user interface (GUI)}

The implementation of animation in our simulation is performed by periodically scheduling a single recurring event called "Ping". A component called "PingThread" simply puts "Ping" events into the event list with deterministic time between occurrences. When the "Ping" event is heard by "Simulation" subcomponent of model component, it updates the locations of mover objects and the "GUIMain" subcomponent of GUI component repaints the icons of mover objects on the map view. A screen shot of a simple implementation of this is in Fig. 8.

This sample screen shot of animation shows a deployment using road (light brown lines) and rail networks (bold black lines) and sea lines (Best viewed in color). A detailed treatment of simple movement and animation in DES is presented in [16].

\subsection{Verification and validation}

Verification and validation were conducted by using appropriate methods explained in [17,18]. To mention a few more specifically, for face validity, we have discussed inputs and outputs of the model and its EGs with potential users of the model and personnel at Transportation Coordination Center of the General Staff. We used assertion checking to verify that the model functioned within its acceptable domain. Incrementally, bottom-up testing was performed, where each individual subcomponent was tested and integrated. Fault (failure) insertion testing was used to test whether the model responded by producing an invalid behavior given the faulty component. During special input testing, we used an arbitrary mixture of minimum and maximum values, and invalid data for the input variables, and tested for potential peculiar situations at the boundary values. In addition, we have tested the validity and behavior of the model under extreme workload and congestion at the load/unload docks and transfer points such as SPOEs, SPODs, etc. Animation also helped in discovering errors during model development.

Furthermore, results of the deployment optimization model developed by Akgün and Tansel [1] were used for verification purposes. Akgün and Tansel used three different networks with number of nodes 13, 19, and 25 and number of arcs 49 , 77 , and 109, respectively. For each network, they generated five problems corresponding to 4, 8, 16, 32, and 64 types of items. Of the 15 problem types generated by Akgün and Tansel, we have used for verification purposes three of them, corresponding to three different network sizes, and 32 item types. We report here only the scenario corresponding to the network with 13 nodes and 49 arcs depicted in Fig. 9.

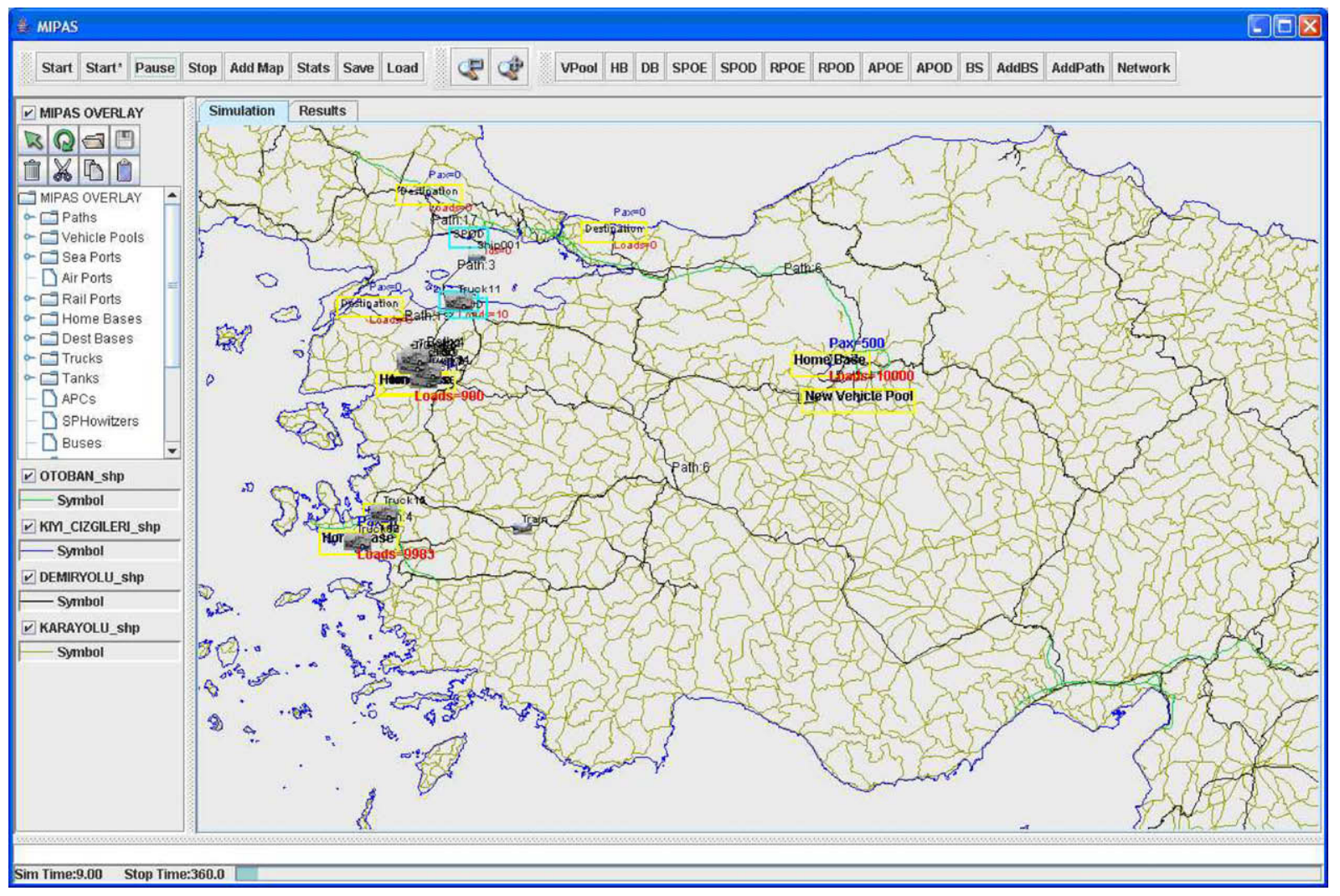

Fig. 8. A sample screen shot of animation showing a deployment using road (light brown lines) and rail networks (bold black lines) and sea lines (best viewed in color). (For interpretation of the references to color in this figure legend, the reader is referred to the web version of this article.) 


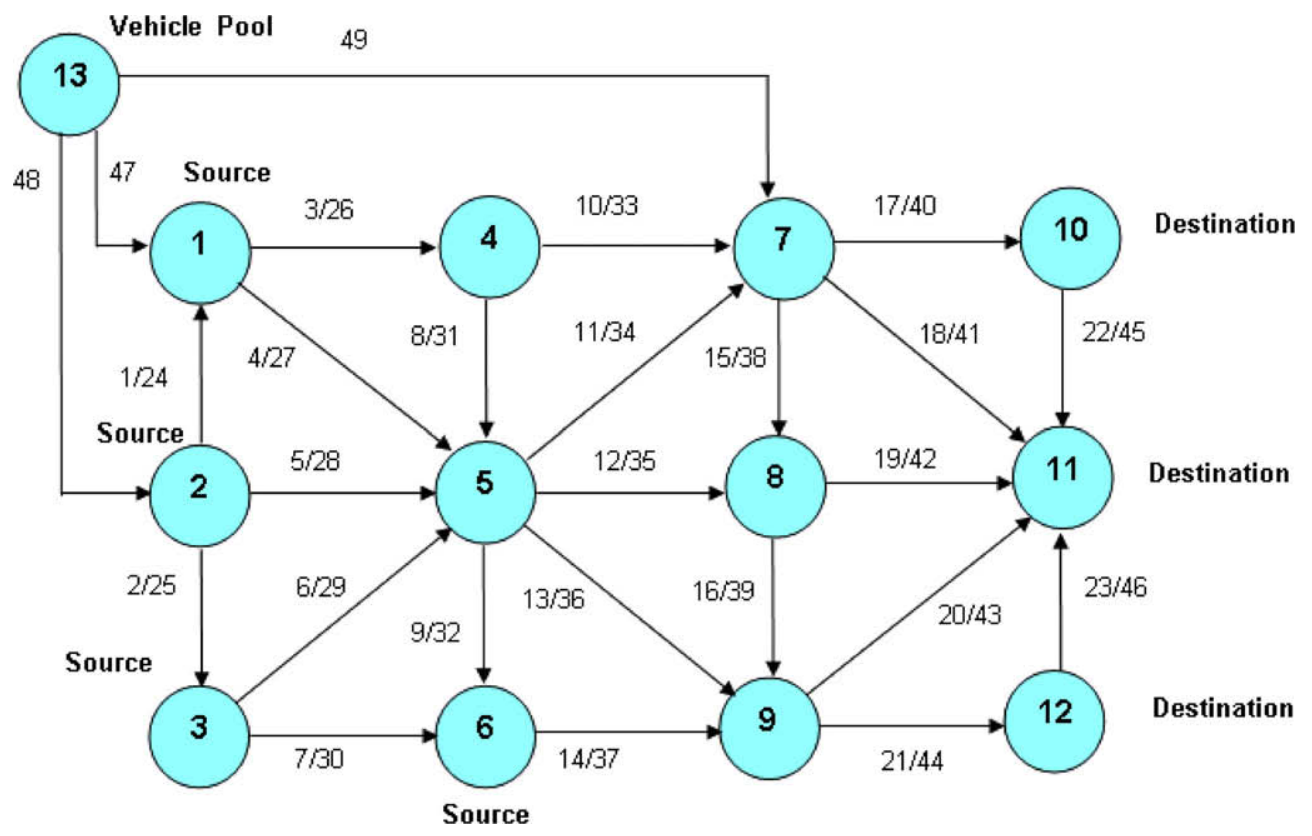

Fig. 9. One of the network models used for verification purposes.

The first set numbers on the arcs are those of the directed arcs depicted in Fig. 9. The second set of numbers belongs to the reverse arcs. In this network, source nodes (home bases) are 1, 2, 3, and 6. Demand nodes (destinations) are 10, 11, and 12. Transshipment nodes are 4, 5, 7, 8, and 9. There is no transfer node (POE or POD). Dummy node (vehicle pool) is 13. It provides transportation assets inorganic to the units and can be regarded as a home base for vehicles. In this scenario, 32 different item types of varying amounts are transported. Item types 1, 9, 17, and 25 are pax. The others are loads of different dimensions and weights. There are six different types of TAs allocated to this scenario. The total number of TAs allocated is 61 vehicles. Load and unload times for each TA is $1 \mathrm{~h}$. For all TAs, the minimum and maximum arrival times at destinations are 80 and $100 \mathrm{~h}$, respectively. The scenario had around 180,000 single variables, 4300 discrete variables and 130,000 equations. The objective was to find the minimum cost deployment plan meeting the time-window deadlines to be at destination. This scenario had only one mode of transportation (land). The scenario was solved to optimality via ILOG CPLEX 9.0 on a $1.5 \mathrm{GHz}$ PIV PC with 1.5 GB RAM. The optimal cost obtained is $\$ 5,727,912.00$. This cost figure includes fixed cost of procurement of TAs and the variable cost of travel per unit time.

A part of the optimal solution of the model that shows movement routes and times of loaded trucks is in Table 2 .

The first line in Table 2 shows that one TA of type 1 departs from source node 1 at time $t=68$, and travels through nodes 5 and 9 to reach the demand node 12 . The first three lines of Table 2 indicate that a total of $18(1+16+1)$ vehicles depart as a convoy from source node 1 at time $t=68$. Upon reaching demand node 12 (at time $t=82$, not shown), these vehicles unload and return to source node 6 . As seen in the last three rows in Table 2, these vehicles in the first three lines of Table 2 that departed at $t=68$ for destination node 12, now depart at $t=90$ from source node 6 to demand node 10 . Thus, in the optimal

Table 2

Optimal numbers, movement routes, and movement times of loaded trucks.

\begin{tabular}{|c|c|c|c|c|c|c|}
\hline Convoy \# & Departure time & Source node & TA \# & TA type & Through nodes & Demand node \\
\hline \multirow[t]{3}{*}{1 (First trip) } & 68 & 1 & 1 & 1 & $5-9$ & 12 \\
\hline & 68 & 1 & 16 & 6 & $5-9$ & 12 \\
\hline & 68 & 1 & 1 & 4 & $5-9$ & 12 \\
\hline \multirow{3}{*}{2 (First trip) } & 68 & 3 & 10 & 3 & $5-7$ & 10 \\
\hline & 68 & 3 & 2 & 4 & $5-7$ & 10 \\
\hline & 68 & 3 & 4 & 6 & $5-7$ & 10 \\
\hline \multirow[t]{3}{*}{2 (Second trip) } & 89 & 2 & 10 & 3 & $5-9$ & 11 \\
\hline & 89 & 2 & 2 & 4 & $5-9$ & 11 \\
\hline & 89 & 2 & 4 & 6 & $5-9$ & 11 \\
\hline \multirow{3}{*}{1 (Second trip) } & 90 & 6 & 1 & 1 & $5-7$ & 10 \\
\hline & 90 & 6 & 1 & 4 & $5-7$ & 10 \\
\hline & 90 & 6 & 16 & 6 & $5-7$ & 10 \\
\hline
\end{tabular}


solution of network in Fig. 9, multiple trips are made from source node 1 to destination node 12 and from source node 6 to destination node 10 by the same set of vehicles shown in the first and last three rows of Table 2 . The remaining rows of Table 2 also depict multiple trips between source node 2 to destination node 11 , and source node 3 to destination node 10 . The optimization results for movement of empty TAs from dummy node (vehicle pool) 13, and after unloading at destination nodes are not included here. The results of the optimization model were simulated using deterministic values used in the optimization model. That is, load and unload times were taken as $1 \mathrm{~h}$ each. Travel times used in arcs of the optimization network were transferred to distance figures using constant travel speeds ( $60 \mathrm{~km} / \mathrm{h}$ ) to be able to physically create the network in the simulation. The cost figure obtained under these deterministic conditions from the simulation was the same as the cost figure obtained from the optimization model.

In addition, simulation results were compared to the historical deployment data obtained from the Scientific Decision Support Center of the General Staff. The results of the comparison were satisfied in terms of validation of our model.

\section{Simulation analysis of a typical real-world case study}

A deployment scenario for deploying four battalions (three mechanized and one armored) during peace-time from northwestern Turkey to the Iraqi border in the southeast is analyzed. The scenario uses land, sea, and rail transportation networks and assets. These four units deploy from three different home bases to three unique destinations. Units C and D are co-located, and Units B and C deploy to the same destination location. The data related to the deployment of each unit and its components are listed in Table 3.

As shown in the line allocated to Unit A in Table 3, Unit A deploys in three components; advance, pax, and cargo parties. The advance party for Unit A has 40 pax, one 2.5-ton truck, one generator, three type 1 boxes, and three type 2 boxes. The pax party for Unit A has 450 personnel. The cargo party for Unit A will deploy by sea. It has 10 pax, 45 armored personnel carriers (APCs), 14 tanks, nine 2.5-ton trucks, ten 5-ton trucks, seven generators, 37 type 1 boxes, and 37 type 2 boxes. Unless otherwise indicated in Table 3, each component deploys by land. The total number of deployed personnel and equipment for Unit A are shown in the line named total. Units B, C, and D deploy in two components each. Their cargo parties deploy by rail as indicated in Table 3.

Table 4 shows the minimum and maximum time requirements for deployed units to be at their designated destination locations. For example, all components of Unit B must deploy not before $120 \mathrm{~h}$ and not after $240 \mathrm{~h}$.

Table 5 shows the initial delay times for each deployment component. Delay times are used to ensure timely and coordinated arrivals at destinations for each deploying unit. For example, Pax component of Unit D departs after $36 \mathrm{~h}$ of simulation time, and its Cargo component departs after $48 \mathrm{~h}$.

The preparation time for all units in this scenario is 10 days ( $240 \mathrm{~h}$ ) and is not listed in Table 5 . The preparation time is also not simulated. The simulation for this scenario is a terminating one with a termination time of $240 \mathrm{~h}$. The transportation assets allocated to this scenario are not listed in detail here. They include one large RoRo ship, four trains with enough and

Table 3

Units listed by their deployment components and major equipment.

\begin{tabular}{|c|c|c|c|c|c|c|c|c|c|}
\hline Unit name & Deployment component & Pax number & APC & Tank & 2.5-ton truck & 5-ton truck & Generator & Box (type 1) & Box (type 2) \\
\hline \multirow[t]{4}{*}{ Unit A } & Total & 500 & 45 & 14 & 10 & 10 & 8 & 40 & 40 \\
\hline & Advance party & 40 & & & 1 & & 1 & 3 & 3 \\
\hline & Pax party & 450 & & & & & & & \\
\hline & Cargo party by sea & 10 & 45 & 14 & 9 & 10 & 7 & 37 & 37 \\
\hline \multirow[t]{3}{*}{ Unit B } & Total & 500 & 45 & 14 & 10 & 10 & 8 & 40 & 40 \\
\hline & Pax party & 450 & & & & & & & \\
\hline & Cargo party by rail & 50 & 45 & 14 & 10 & 10 & 8 & 40 & 40 \\
\hline \multirow[t]{3}{*}{ Unit C } & Total & 500 & 45 & 14 & 10 & 10 & 8 & 40 & 40 \\
\hline & Pax party & 450 & & & & & & & \\
\hline & Cargo party by rail & 50 & 45 & 14 & 10 & 10 & 8 & 40 & 40 \\
\hline \multirow[t]{3}{*}{ Unit D } & Total & 500 & 14 & 45 & 10 & 10 & 8 & 40 & 40 \\
\hline & Pax party & 450 & & & & & & & \\
\hline & Cargo party by rail & 50 & 14 & 45 & 10 & 10 & 8 & 40 & 40 \\
\hline
\end{tabular}

Table 4

Time windows for units to be deployed.

\begin{tabular}{lll}
\hline Unit name & Min time (hours - days) & Max time (hours - days) \\
\hline Unit A & $(0-0)$ & $(120-5)$ \\
Unit B & $(120-5)$ & $(240-10)$ \\
Unit C & $(120-5)$ & $(240-10)$ \\
Unit D & $(48-2)$ & $(168-7)$ \\
\hline
\end{tabular}


Table 5

Initial delay times for each deployment component.

\begin{tabular}{ll}
\hline Unit name & Deployment component \\
\hline Unit A & Advance party \\
& $\begin{array}{l}\text { Pax party } \\
\text { Cargo party by sea }\end{array}$ \\
Unit B & Pax party delay (h) \\
& Cargo party by rail \\
Unit C & Pax party \\
Unit D & Cargo party by rail \\
& Pax party \\
\hline
\end{tabular}

appropriate rail cars, trucks, and tank carriers (one tank carrier can carry a single tank or 2 APCs). The armored vehicles must be carried by rail or sea to distances over $300 \mathrm{~km}$. In this scenario, they deploy over $300 \mathrm{~km}$. Other restrictions used in the simulation, such as the storage capacities of SPOE, SPOD, RPOE, and RPOD, are not presented here.

\subsection{Results}

We adjust the sample size by setting the number of replications. To achieve the desired accuracy of $90 \%$ relative precision ( $10 \%$ error), we first ran the model for 5 replications for a run-length of $240 \mathrm{~h}$. Using our performance measure of percentage of average on-time arrivals at each unit's destination, we calculated point and interval estimates. For each unit, the halfwidth as a measure of accuracy varied. In order to achieve the desired accuracy in the worst case, we used the maximum half-width for all units for the given run-length and decided that 10 runs would suffice.

Yet, since run-time is not expensive in our case, we ran the simulation for 60 replications (around $25 \mathrm{~min}$ ) on a $1.6 \mathrm{GHz}$ Pentium(R) M PC with 512 MB RAM to see the model's behavior. Our performance measure was the percentage of average number of TAs from each of the four units that arrived in their given time windows at their respective destinations. This performance measure was met for each unit. However, there were different numbers of vehicle breakdowns that lasted varying amounts of time. Even though all TAs from 4 units arrived during their given time windows, some had to deal with delays due to maintenance problems. To be more specific, on average, Unit A had two minor breakdowns per run. Unit B had one minor breakdown every four runs. Unit C had 9 breakdowns per 5 runs, and 17 medium breakdowns per 20 runs. Unit D had one minor breakdown per run, one medium breakdown per 5 runs, and one major breakdown per 4 runs.

That is, all deployed units arrived at their respective destinations with some maintenance problems. This is common for any military deployment scenario, yet it does not provide much insight other than to have spare equipment and personnel to fix broken down vehicles. In order to see how this plan would behave in an emergency situation, such as a quickly escalating military situation or a natural disaster, we decided to reduce allowed maximum arrival times (Tmax) at each unit's respective destinations by 12-h increments and ran the simulation for each case. Even though a replication number of 10 is sufficient, we used 15 replications for each case just to be on the safe side. We made a total of $15 \times 12=180$ replications. We assumed that since the situation is urgent, the initial delay figures depicted in Table 4 are zero. Likewise, the allowed minimum arrivals times at each unit's respective destination are taken as zero.

The results are displayed for each unit. These figures represent the robustness of a peace-time deployment scenario under urgent circumstances. For Unit A, all deployment components arrived on-time for the peace-time scenario with Tmax $=120 \mathrm{~h}$. Fig. 10 depicts that the percentage of average on-time arrivals for Unit A after Tmax $=96 \mathrm{~h}$ drops below $20 \%$. This is unacceptable by any military standard.

Units B and C arrive at the same destination, and thus are considered together. For Units B and C, there is no change of average on-time arrivals in the range Tmax is considered. That is, this deployment scenario is robust for Units B and C. Fig. 11

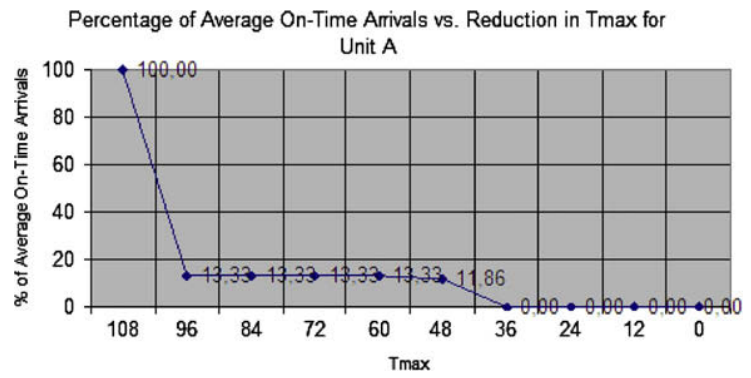

Fig. 10. Percentage of average on-time arrivals vs. reduction in Tmax by $12 \mathrm{~h}$ for Unit A. 


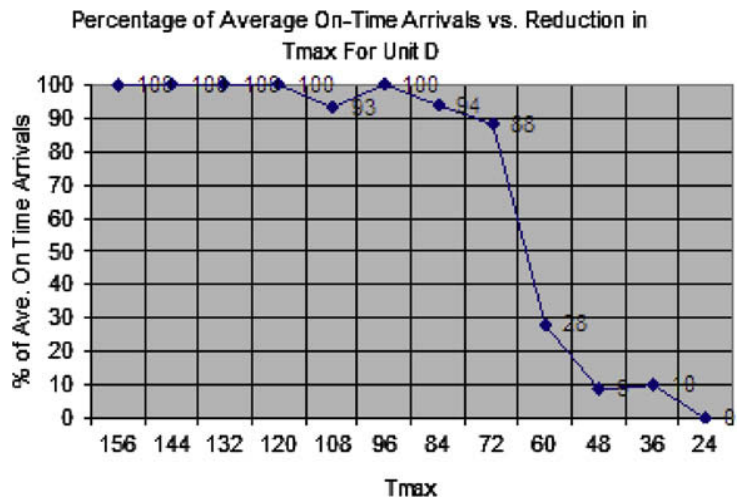

Fig. 11. Percentage of average on-time arrivals vs. reduction in Tmax by $12 \mathrm{~h}$ for Unit $\mathrm{D}$.

shows that the danger zone for Unit D lies between $T \max =72 \mathrm{~h}$ and $60 \mathrm{~h}$ as the percentage of average on-time arrivals drops from $88 \%$ to $28 \%$. Twenty-eight percentage is certainly unacceptable.

These are very critical information as to what would happen if an existing plan had to be modified accordingly as unexpected events unfold. A military mission cannot be accomplished and lives could be lost if military units cannot arrive at their designated destinations in a timely manner according to their plans. Our simulation proves to be an invaluable tool to create, and to determine the applicability and robustness of existing plans in a relatively short time.

\section{Conclusions}

In this paper, we have introduced a logistics and transportation simulation developed for use in the military DPP. We applied, for the first time, the EG methodology and LEGO framework to create a multi-modal discrete-event simulation model of the DPP. EGs and LEGOs provided a simple yet powerful and elegant way of representing DES model of deployment, and enabled easy creation of component-based models of a real-world military problem. The medium-resolution used allowed us estimate whether a given plan of deployment will go as intended, and determine prospective problem areas in a relatively short time compared to other existing simulations because of the absence of the need to use several models of differing resolutions in succession. The short run times achieved demonstrated this. The very accurate and detailed GIS data, and the detailed data used in modeling of entities, resources and military equipment in the simulation permitted us not to exchange reality in favor of shorter run times. We had to extend Simkit by writing additional Java classes that are specific to military deployment. The component-based approach adopted in development of our simulation model enables us to easily integrate future additions to our model. These additions may be detailed modeling of infrastructure and resources at transfer points. Our model is generic enough to be used in commercial logistics applications after some problem-specific modifications. Finally, we have simulated a real-world case study to see its robustness under urgent situations. Our simulation provided valuable insights as to when and what percentage of units would be at their designated destinations if the original plan had to be modified for more urgent deployment of military units. This is invaluable information for a commander since a military mission cannot be accomplished and lives could be lost if military units can not arrive at their designated destinations in a timely manner according to their plans.

\section{Acknowledgements}

We thank Mr. Erhan Çınar and Mr. Murat Durmaz of BilgiGIS Ltd. of Ankara, Turkey, for sharing their expertise in GIS, providing guidance and help in integrating their GIS software, GeoKIT, with our simulation model. We thank Prof. Arnold Buss of Naval Postgraduate School for his help and insights on our questions about Simkit. We appreciate the comments and suggestions of the anonymous referees which helped improve our paper. A preliminary and shorter version of this paper has been published at the Proceedings of Winter Simulation Conference in December 2007 [19].

\section{References}

[1] İ. Akgün, B.Ç. Tansel, Optimization of transportation requirements in the deployment of military units, Computers and Operations Research 34 (4) (2007) $1158-1176$.

[2] A. Boukhtouta, A. Bedrouni, J. Berger, F. Bouak, A. Guitouni, A survey of military planning systems, <http://www.dodccrp.org/events/9th_ICCRTS/CD/ papers/096.pdf>, 2004 (accessed 15.12.07).

[3] K. McKinzie, W.J. Barnes, A review of strategic mobility models supporting the defense transportation system, <http://www.me.utexas.edu/ barnes/ research/files/update_06_2003/RSMMSDTS.pdf>, 2003 (accessed 15.12.06).

[4] G.N. Heal, I.K. Garnett, Allied Deployment and Movement System (ADAMS) Version 3.0 Tutorial, Technical Note 669, NATO Consultation, Command and Control Agency, The Hague, September 2001.

[5] C.N.V. Groningen, D. Blachowicz, M.D. Braun, R.J. Love, K.L. Simunich, M.A. Widing, Modeling military deployment in theaters of operations - balancing deployment alternatives, <http://www.dis.anl.gov/temps/LogisticsSpectrum.ELIST.MergedFigures.8Aug05.pdf>, 2005 (accessed 15.12.06). 
[6] L.C. Krause, J. David Parsons, Tactical logistics and distribution system (TLOADS) simulation, in: Proceedings of the 1999 Winter Simulation Conference, 1999.

[7] D.L. Howard, M.J. Bragen, J.F. Burke Jr., R.J. Love, PORTSIM 5: modeling from a Seaport Level, Mathematical and Computer Modeling (2004).

[8] J.F. Burke, R.J. Love, C.M. Macal, D.L. Howard, J. Jackson, Modeling force deployment from army installations using the transportation system capability (TRANSCAP) model, <http://www.osti.gov/bridge/servlets/purl/757530-YBfenI/webviewable/757530.pdf> (accessed 15.12.06).

[9] SIMULOGS (Simulation of Logistics Systems) <http://ms.ie.org/SIW_LOG/Log_Federates/SIMULOGS.ppt> (accessed 15.12.06).

[10] D. Goldsman, S. Pernet, K. Kang, Simulation of transportation logistics, in: E. Yücesan, C.-H. Chen, J.L. Snowdon, J.M. Charnes (Eds.), Proceedings of the 2002 Winter Simulation Conference, 2002.

[11] L. Schruben, Simulation modeling with event graphs, Communications of the ACM 26 (11) (1983) 957-963.

[12] R.G. Sargent, Event graph modeling for simulation with an application to flexible manufacturing systems, Management Science 34 (10) (1988) 12311251.

[13] A.H. Buss, Basic event graph modeling, Simulation News Europe 31 (2001) 1-6.

[14] A.H. Buss, P.J. Sanchez, Building complex models with LEGOS (listener event graph objects), in: E. Yücesan, C.-H. Chen, J.L. Snowdon, J.M. Charnes (Eds.), Proceedings of the 2002 Winter Simulation Conference, Institute of Electrical and Electronics Engineers, Piscataway, New Jersey, 2002.

[15] A.H. Buss, Component-based simulation modeling using Simkit, in: E. Yücesan, C.-H. Chen, J.L. Snowdown, J.M. Charnes (Eds.), Proceedings of the 2002 Winter Simulation Conference, Institute of Electrical and Electronics Engineers, Piscataway, New Jersey, 2002.

[16] A.H. Buss, P.J. Sanchez, Simple movement and detection in discrete event simulation, in: M.E. Kuhl, N.M. Steiger, F.B. Armstrong, J.A. Joines (Eds.), Proceedings of the 2005 Winter Simulation Conference, Institute of Electrical and Electronics Engineers, Piscataway, New Jersey, 2005.

[17] R.G. Sargent, Some approaches and paradigms for verifying and validating simulation models. in: B.A. Peters, J.S. Smith, D.J. Mederios, M.W. Rohrer (Eds.), Proceedings of the 2001 Winter Simulation Conference, Institute of Electrical and Electronics Engineers, Piscataway, New Jersey, 2001.

[18] O. Balcı, Verification Validation and Testing, Handbook of Simulation, John Wiley \& Sons, Inc., 1998.

[19] U.Z. Yıldırım, İ. Sabuncuoğlu, B.Ç. Tansel, A simulation model for military deployment, in: S.G. Henderson, B. Biller, M.-H. Hsieh, J. Shortle, J.D. Tew, R.R. Barton (Eds.), Proceedings of the 2007 Winter Simulation Conference, 2007. 